\title{
Factores que impiden el desarrollo de equipos de trabajo en la Facultad de Ciencias Administrativas"
}

\author{
(*) Lic. Luis Flores Hidalgo \\ e-mail:d180018@unmsm.edu.pe
}

\section{RESUMEN}

El presente estudio, define el problema de la siguiente manera: " ¿ Cuáles son los factores de los grupos de trabajo universitario que impiden el desarrollo de equipos de trabajo en la Facultad de Ciencias Administrativas, de la Universidad Nacional Mayor de San Marcos y en el semestre 99-Il ? "

El método que se utilizó para la ejecución del presente estudio fue el descriptivo-analítico. El estudio comprendió las bases 97 y 98 de las Escuelas Académico Profesionales de Administración de Turismo, Administración de Negocios Internacionales y Administración de la Facultad de Ciencias Administrativas de la Universidad Nacional Mayor de San Marcos. La población está integrada por 735 alumnos. Se seleccionó el tipo de muestreo probabilístico aleatorio simple y la muestra comprende en términos de proporciones el $11 \%$ del total de la población, con un margen de error del $5 \%$. Para la recolección de los datos se utilizó como método la encuesta, siendo el instrumento el cuestionario. Se desarrolló una encuesta piloto con la finalidad de determinar errores en la formulación de las preguntas y alternativas.

\section{Resultados mas importantes}

1. El número ideal de integrantes para trabajar en grupo es cuatro.

2. Las cuatro actitudes más importantes en el desarrollo de las reuniones grupales:

Comunicar sus ideas, Respetar las ideas contrarias, Escuchar a todos los integrantes e, Idéntificar $y$ reconocer las habilidades de los miembros.

3. Los alumnos perciben que la infraestructura de la facultad no facilita el desarrollo de trabajos grupales.

4. Las sugerencias para mejorar los trabajos de grupo
Ambientes para trabajos grupales.
O Proporción de Tecnología/Equipos y
O Mejora de la Biblioteca

5. Un porcentaje representativo declaró no tener una metodología para las reuniones de trabajo, indicando que solo a veces se programan las actividades en cada reunión grupal.

6. Entre los factores más importantes que impiden el desarrollo efectivo del grupo se encuentran:

O Insuficiente comunicación.

O Infraestructura inadecuada.

O Poca motivación.

O Carencia de responsabilidad.

7. Mas de la mitad de los encuestados reconoce que su grupo no esta actuando como un equipo.

\section{Principales conclusiones:}

1. No existe un compromiso genuino de los alumnos con sus grupos de trabajo, lo que impide que se desarrollen como equipos de trabajo.

2. Los factores que favorecen el trabajo en grupo son: ambiente para trabajos grupales, proporción de tecnología, equipos necesarios y mejora de la biblioteca.

3. Los alumnos poseen habilidades deseables para desarrollarse como miembros de un equipo de trabajo, porque la mayoría de sus miembros:

O Coordinan sus actividades.

O Comunican sus ideas.

O Escuchan a todos sus integrantes.

O Reconocen sus limitaciones y habilidades ante el grupo.

- Reconocen que existen deficiencias en el proceso de planificación de las reuniones de trabajo.

4. Los estudiantes reconocen que una reunión de trabajo grupal no es planificada y sujeta a una estricta agenda.

5. El número ideal para un grupo de trabajo es de 
cuatro, excepcionalmente cinco integrantes.

6. Los alumnos reconocen que el trabajo en grupo es importante para su formación profesional.

7. Entre los tres factores mas importantes que impiden el desarrollo efectivo del grupo se encuentran: la comunicación, no poder contar con una infraestructura adecuada y motivación.

\section{INTRODUCCIÓN}

Un equipo no es un grupo de personas trabajando juntas. Tampoco las características particulares de cada uno de sus miembros tiene porque determinar los resultados de la labor desarrollada en grupo. Por lo tanto: ¿Qué es un verdadero equipo?. ¿Cómo pueden saber los docentes cuándo tiene sentido trabajar en equipo y cómo se puede asegurar el éxito de su labor?. El presente trabajo responde a estas cuestiones siendo los objetivos del mismo:

O Describir sí los grupos de trabajo planifican sus actividades.

O Identificar el número ideal de integrantes para trabajar en grupo.

O Averiguar cuales son las actitudes más importantes en el desarrollo de las reuniones grupales.

O Identificar sugerencias para mejorar los trabajos de grupo, según opinión de los respondientes.

O Determinar los factores de los grupos de trabajo que impiden el desarrollo de equipos en la Facultad de Ciencias Administrativas de la UNMSM en el semestre 99-II.

\section{Justificación del estudio}

El presente trabajo de investigación responde a la necesidad de contribuir con la formación de profesionales que se desempeñen eficientemente dentro de los equipos de trabajo organizacional y además estén en capacidad de formarlos y liderarlos, lo cual implica una constante ejercitación por parte de los alumnos y un constante monitoreo por parte de la plana docente desde las aulas universitarias. Se pretende dar sugerencias que permitan superar las deficiencias de los actuales grupos de trabajo; así como también, puedan ser aplicados en los grupos de trabajo de los alumnos de los primeros ciclos, para que tengan un comportamiento de equipo.
La población está distribuida de la siguiente forma:

\begin{tabular}{|l|c|c|c|}
\hline \multicolumn{1}{|c|}{ ESCUELA } & BASE 97 & BASE 98 & TOTAL \\
\hline Administración & 178 & 209 & 387 \\
\hline Adm. deTurismo & 85 & 92 & 174 \\
\hline Adm. de Negocios Internacionales & 70 & 104 & 174 \\
\hline
\end{tabular}

\section{MARCO TEORICO}

Douglas McGregor identificó las características de un equipo de trabajo eficaz:

1. El ambiente tiende a ser informal, cómodo y relajado, entonces las personas se sienten involucradas e interesadas.

2. Hay mucha discusión y prácticamente todos participan, pero se mantiene dentro de las tareas del grupo, si la discusión se sale del tema, alguien la traerá de vuelta en poco tiempo.

3. La tarea o el objetivo del grupo es bien entendido $y$ aceptado por los miembros. En algún momento habrá discusión libre del objetivo, hasta que se formule de tal modo que los miembros del grupo puedan comprometerse a ello.

4. Los miembros se escuchan unos a otros y las ideas son respetadas.

5. No se reprimen los desacuerdos ni son anulados con una acción prematura del grupo. Se estudian las razones de las objeciones con cuidado y el grupo busca resolverlas en vez de dominar al disidente.

6. La mayor parte de las decisiones se toman por consenso, en el cual es evidente que todos, en general, están de acuerdo y dispuestos a adherirse.

7. La crítica es frecuente, franca y relativamente cómoda. La crítica tiene un enfoque constructivo orientado a remover un obstáculo al que se enfrenta el grupo y que impide que el trabajo se realice.

8. Cuando se decide realizar una acción, se hacen asignaciones claras y estas son aceptadas.

9. El jefe del grupo no lo domina, ni tampoco el grupo muestra diferencia hacia él. La cuestión no es quién controla, sino como llevar a cabo el trabajo.

10. El grupo esta consciente de sus propias operaciones. Con frecuencia, se detiene para estudiar cómo lo está haciendo o qué puede estar interfiriendo con su operación. El problema puede ser una cuestión de procedimiento, o puede tratarse de un individuo cuyo comportamiento está obstaculizando el cumplimiento de los objetivos del grupo. Sea lo que sea se discute abiertamente hasta encontrar la solución. ${ }^{1}$ 
Rensis Likert en su texto New Patterns of Managernent, desarrollaba su idea de las organizaciones como una serie de grupos entrelazados, siendo el gerente el "punto de unión". Likert consideró que los gerentes debían aprender a hacer frente a la totalidad del personal bajo su dirección y no sólo dirigir a los individuos uno por uno. La forma ideal de dirección de Likert (Sistema 4) se denomina dirección de "grupo participativo", en este sistema el investigador incluyó a los gerentes exitosos.

Las ideas, metas $y$ actitudes no se congelan si los miembros son capaces de ejercer influencia, uno sobrt otros, de manera continua.

En un grupo altamente eficaz existen firmes motivaciones para intentar ejercer influencia sobre otros miembros y también para aceptar la influencia de ellos. Esto se aplica a todas las actividades del grupo: asuntos técnicos, métodos, problemas organizacionales, relaciones interpersonales y procesos del grupo.

Los mejores equipos dedican gran tiempo y esfuerzo a explorar, dar forma y ponerse de acuerdo, de manera que acaben sintiendo como propio -tanto individual como colectivamente- el propósito de que se trate. Este tipo de actividad, directamente relacionada con el propósito. Continúa desarrollándose durante todo el tiempo que el equipo funciona como tal. Por el contrario, los equipos que fracasan no suelen crear este propósito compartido por todos. Por la razón que sea -porque pierden de vista los resultados. Por falta de esfuerzo o de liderazgo- no convergen en torno a una aspiración que supone un reto importante. 2

Blake y Mouton han usado ampliamente la analogía del equipo al escribir su "Dirección 9,9" :

Como es probable que el equipo de trabajo se utilice para referirse a cualquier conjunto de individuos que cooperen para lograr un solo resultado general, se puede preguntar si la acción de equipo del tipo que se está describiendo conduce a presiones de conformidad, lo cual disminuye la individualidad $y$ reprime la independencia en el esfuerzo. La respuesta es que lo opuesto es la verdad. La verdadera acción de equipo se parece más a una situación en el fútbol, donde la división del esfuerzo se enlaza en un solo resultado cbordinado. donde el total es más, y diferente, que la suma de sus partes individuales. Aquí, existen un conjunto común de señales, basadas en la comprensión, lo cual dicta la acción. Una división de actividad individual estrechamente unida en combinación con un esfuerzo interdependiente es el patrón 9.9 para integrar el esfuerzo individual con el esfuerzo colectivo.

Estos autores señalan que un buen equipo tiene especialización en la tarea y división de trabajo.

Cada una de las personas carga una parte del trabajo total, con un $100 \%$ de responsabilidad por el éxito del grupo... Además tiene una serie común de estrategias que se adaptan a diversas situaciones y señales que son bien entendidas e incluye:

1. Acción del equipo basada en el esfuerzo sincronizado de todos.

2. Acción de pareja; es decir, basándose en el esfuerzo combinado del director técnico $y$ el mariscal de campo, el mariscal y el centro, etc.

3. El esfuerzo individual; es decir correr a través del campo, interceptación de un lanzamiento, etc.

Eł baloncesto es algo muy diferente al fútbol. En este caso, el equipo es pequeño, todos juegan en un solo equipo. Cada uno de los jugadores está involucrado en todos los aspectos del partido, como ofensiva $y$ como defensiva, y todos deben hacer pases, correr y lanzar la pelota al cesto. Cuando entra un sustituto, todos deben jugar con la nueva persona.

Por otra parte, el director técnico del equipo de fútbol necesita desarrollar una estrategia de juego y también debe tomar algunas decisiones tácticas. El director técnico del equipo de baloncesto trabajará con todo el equipo, puesto que es pequeño, mientras que el director técnico del equipo de fútbol necesita un grupo de ayudantes que manejen diversos grupos, el director técnico principal del equipo de fútbol tiene la responsabilidad de estructurar el grupo de directores técnicos asistentes en su propio equipo. ${ }^{3}$

Modelo para la conformación de equipos.

El modelo para la conformación de equipos de alto rendimiento está diseñado para mantener una visión sistemática de la organización y está compuesto por los siguientes elementos:

I. Focalizar la acción del esfuerzo del equipo de alto rendimiento.

El proceso de formular el futuro de cada subsistema o equipo efectivo en coherencia con el sistema empresa (interrelación) y en congruencia con los 
demás subsistemas (Interdependencia) comprende los siguientes aspectos:

1. La Visión compartida.

Una de las primeras medidas que un equipo de alto rendimiento debe realizar es visualizar lo que pretende lograr en forma coherente con la visión compartida de la empresa y en congruencia con los demás subsistemas.

2. La misión.

Es la contribución única que el equipo elige para hacer que el subsistema al que pertenece se mueva hacia la visión. Tambien se le denomina finalidad y es la concepción implícita del "por qué" del subsistema. La misión debe ser compromiso por todos compartido.

3. Código de valores y principios guía del equipo. Los valores y creencias deben ser:

- Significativos: "Llegar al corazón de la gente".

- Formularse en forma explícita y breve.

- Duraderos y alcanzables.

II. Escenario de actuación de equipos de alto rendimiento-sistema cooperativo.

La preparación de este escenario implica:

1. Rediseño de los procesos o de la forma como se hará el trabajo.

2. La configuración estructural o definición de la forma en que se agruparán las personas.

3. El rediseño de los puestos de trabajo. Un puesto de trabajo bien diseñado es aquel que tiene autoridad para resolver por lo menos el $80 \%$ de las situaciones "anormales" que se presentan. La definición del puesto se hace considerando el aporte de conocimiento $y$ habilidad al proceso y a diferentes procesos, y no a la "especialización".

4. Tomar en cuenta las nuevas medidas de valor del cliente:

- Tiempo de ciclo de los procesos (velocidad)

- Costo competitivo (eliminar los extracostos)

- Calidad de los productos con categoría mundial

- Servicio real y perceptivo para el cliente (valor agregado incorporado)

III. Conocimiento y habilidades para la actuación en equipos de alto rendimiento.Para crear el clima que facilite la sinergia, el equipo debe desarrollar los paradigmas de:
1. Actitud gano/ganas.

Significa que los acuerdos o soluciones son mutuamente benéficos, satisfactorios, generando un escenario cooperativo. no competitivo.

2. Comprender antes de ser comprendido.

Escuchar con empatía, lo que significa escuchar a cada persona colocándose en sus "zapatos" para poder comprender lo que está pensando y lo que está sintiendo. Comprender requiere respeto. consideración y valentía, implica que el equipo de alto rendimiento logre una comunicación madura y efectiva.

3. Valorando las diferencias.

Cuando se afirma que "el todo es mayor que la suma de las partes", se está hablando de sinergia. La sinergia implica respetar y valorar las diferencias individuales, aspectos en la conformación y mantenimiento de un equipo efectivo.

El equipo logra a través de la interacción de los miembros resultados que no podían ser logrados si cada persona trabajara individualmente. ${ }^{4}$

\section{Pasos para fomentar la responsabilidad compartida}

1. Asigne tareas que exijan a los integrantes trabajar juntos cooperando para controlar sus esfuerzos.

2. Cree oportunidad para que las personas se ayuden entre sí.

3. Ayuda a cada miembro a reconocer sus propias capacidades y limitaciones, así como las de los demás.

4. Establezca un sistema de recompensas que genere mayor interés por alcanzar resultados de conjunto en vez de un sistema de premios de tipo individual.

5. Haga que cada persona asuma la responsabilidad de una parte del trabajo que conozca como se relaciona sus tarea con la de los demás para lograr su meta.

\section{OCHO ATRIBUTOS DE LOS EQUIPOS DE ALTO RENDIMIENTO}

1. Liderazgo participativo, crea interdependencia dando fuerza , liderando y sirviendo a otros.

2. Responsabilidad compartida, establece un medio en el cual todos los miembros del equipo se sienten tan responsables como el gerente por la eficiencia de la 
unidad de trabajo.

3. Comunidad de propósito, se tiene un sentido de propósito común en cuanto al porque de la existencia del equipo y su función.

4. Buena comunicación, crea un clima de confianza y de comunicación abierta y franca.

5. La mira en el futuro, para ver el cambio como una oportunidad de crecimiento.

6. Concentración en la tarea, se mantienen reuniones centradas en los resultados.

7. Talentos creativos, los talentos y la creatividad individuales están al servicio del trabajo.

8. Respuesta rápida, en la identificación y el aprovechamiento de las oportunidades.
Hay que señalar, igualmente, que cualquier grupo de personas que trabajen juntas no constituyen un equipo. Los comités, consejos y grupos de trabajo no son necesariamente equipos. Todo el personal de cualquier organización compleja y de gran tamaño no constituye jamás un equipo, sin embargo; cuántas veces se utiliza ese tópico.

Un equipo es un conjunto de personas -pocas- con capacidades complementarias, comprometidas con un proposito común, con un conjunto de objetivos en cuanto a resultados y con un enfoque de todo lo cual se consideran conjuntemente responsables.

\section{Cualquier grupo no constituye un equipo. ¿Cuál es la diferencia?}

Grupo

- Tiene un líder fuerte y centrado en la tarea de que se trate.

- La responsabilidad es de cada individuo.

- El propósito del grupo es el mismo que el del resto de la organización.

- El producto del trabajo se genera de forma individual.

- Celebran reuniones que resultan eficaces.

- La eficacia se mide de forma indirecta, mediante los efectos que se producen en otros aspectos (por ej. resultados financieros del negocio).

- Se discute, se decide y se delega.
Equipo

- El liderazgo es compartido por varios.

- La responsabilidad es tanto individual como conjunta.

- El propósito es específico del equipo, que es quien lo consigue

- Se generan productos que son fruto del trabajo colectivo.

- Se fomentan las discusiones abiertas y las reuniones cuyo objetivo es la resolución de problemas de una manera activa.

- Los resultados se miden de forma directa mediante la evạluación del producto del trabajo colectivo.

- Se discute, se decide y se trabaja conjuntamente.

\section{Existe una gran diferencia entre "el jefe me considera responsable" y "nosotros nos consideramos responsables".}




\section{METODOLOGÍA}

\section{Método}

El método utilizado fue el analítico, tipo encuesta.

\section{Descripción del área}

El presente estudio comprendió las bases 97 y 98 de las Escuelas Académico Profesionales de Administración de Turismo, Administración de Negocios Internacionales y Administración de la Facultad de Ciencias Administrativas de la Universidad Nacional Mayor de San Marcos.

Se consideró dicha área de estudio, debido a que las unidades de observación ya han tenido experiencia en trabajos de grupo; y que los resultados del trabajo de investigación están orientados a tomar medidas correctivas que faciliten su desarrollo como equipos de trabajo.

\section{Población y Muestra.}

\section{Población}

La población consta de 735 alumnos, distribuidos de la siguiente manera:

\begin{tabular}{|l|c|c|c|}
\hline \multicolumn{1}{|c|}{ ESCUELA : } & BASE 97 & BASE 98 & TOTAL \\
\hline Administración & 178 & 209 & 387 \\
\hline Adm. de Turismo & 82 & 92 & 174 \\
\hline Adm. de Negocios intemacionales & 70 & 104 & 174 \\
\hline
\end{tabular}

\section{Muestreo}

Se seleccionó el tipo de muestreo probabilístico aleatorio simple, ya que dio igual oportunidad a toda la población de ser parte de la muestra.

\section{Muestra}

La muestra comprende en términos de proporciones el $11 \%$ del total de la población, con un margen de error del. $5 \%$.

\section{Instrumento de recolección de Datos}

Para la recolección de los datos se utilizó como instrumento el cuestionario.

Se desarrolló una encuesta piloto, con la finalidad de determinar errores en la formulación de las preguntas y alternativas.

\section{Hipótesis}

1. El compromiso genuino de los alumnos con el grupo de trabajo facilitará su participación en los equipo de trabajo.

2. La existencia de un ambiente apropiado, contribuirá a que los grupos de trabajo se desarrollen como equipos.

3. El número ideal de integrantes para trabajar en grupo es cinco.

4. Los factores más importantes que favorecen el trabajo en grupo son:

- Ambiente apropiado.

- Contar con tecnología y equipos de punta.

- Contar con una biblioteca especializada.

- Asesoramiento.

5. Los factores que impiden el desarrollo efectivo de los grupos son:

Infraestructura inadecuada.

- Falta de motivación.

- Falta de objetivos.

- Conformismo.

- Poca responsabilidad.

- Comunicación insuficiente

\section{Variables Indicadores}

- Grupo de trabajo. - Número ideal de integrantes.

- Actitudes - Comportamiento en el desarrollo de las reuniones grupales.

- Planificación. - Plan para trabajar en grupo.

- Grupo ineficiente. - Factores que impiden el desarrollo efectivo del grupo.

\section{RESULTADOS}

1. El número ideal de integrantes para trabajar en grupo es cuatro y le sigue en grado de importancia cinco.

2. En la conformación de grupos el objetivo más importante ha sido obtener notas satisfactoria y satisfacción académica grupal. 
3. No todos los miembros del grupo comparten un mismo objetivo al momento de desarrollar un trabajo de investigación.

4. Las cuatro actitudes más importantes en el desarrollo de las reuniones grupales son:

O Comunicar sus ideas

O Respetar las ideas contrarias

O Escuchar a todos los integrantes

O Identificar y reconocer las habilidades de los miembros

5. Los estudiantes reconocen que el trabajo en grupo es importante en su formación profesional.

6. Los estudiantes reconocen la existencia de un jefe de grupo (lider) que orienta las acciones del grupo.

7. Los respondientes manifestaron haber experimentado mejoras en la calidad de sus trabajos grupales.

8. Al momento de desarrollar un trabajo en grupo, los alumnos lo dividen y trabajan individualmente.

9. Ocasionalmente el profesor muestra algún interés en el desarrollo de trabajos grupales.

10. Los alumnos perciben que la infraestructura de la facultad no facilita el desarrollo de trabajos grupales.

11. Las sugerencias para mejorar los trabajos de grupo son:

O Ambientes para trabajos grupales.

O Tecnología/Equipos

O Mejorar la Biblioteca

12. Los alumnos contestaron que normalmente trabajan en grupo de cuatro, en segundo lugar de cinco y un poco lejos de tres.

13. Un porcentaje representativo puso en evidencia no tener una metodología para las reuniones de trabajo.

14. Los integrantes de un grupo sólo a veces sacrificarían sus metas personales por del grupo.

15. Solo a veces se programan las actividades en cada reunión grupal.
16. No existe un liderazgo absoluto y generalmente las actividades del grupo son coordinadas por más de uno.

17. Los entrevistados reconocieron ante el grupo sus habilidades y por lo tanto su contribución al mismo.

18. Existe un alto grado de identificación con su grupo de trabajo.

19. La mitad de los entrevistados consideran que su grupo no actúa como un equipo.

20. Los respondientes manifestaron que la visión y misión compartida son los principales factores que influyen en el desarrollo de equipos, siguiendole en orden de importancia el ambiente de trabajo.

21. Entre los factores más importantes que impiden el desarrollo efectivo del grupo se encuentran:

O Comunicación

O Infraestructura inadecuada

O Motivación

O Responsabilidad

\section{CONCLUSIONES}

1. No existe un compromiso genuino de los alumnos con sus grupos de trabajo, lo que impide que se desarrollen como equipos de trabajo. Sin embargo existe interés e identificación con los miembros del grupo, ya que el factor principal de la conformación de los grupos es la amistad y/o afinidad de sus miembros.

2. Para el desarrollo de trabajo en equipos se debe proporcionar un ambiente de estudio adecuado.

3. El número ideal para un grupo de trabajo está entre cuatro $y$ cinco integrantes.

4. Entre los factores (en orden de prioridad) que favorecen el trabajo en grupo son:

O Ambientes para trabajos grupales.

O Proporcionar la tecnología y equipos necesarios. O Mejorar la biblioteca.

5. Entre los factores en (orden de prioridad) que impiden el desarrollo efectivo de los grupos tenemos: 
O Insuficiente comunicación.

O Infraestructura inadecuada.

O Poca motivación.

O Carencia de responsabilidad.

6. Existe bajo nivel de interés de los profesores, tanto por la tarea del trabajo grupal, como por el desarrollo del grupo para que este llegue a ser un equipo.

7. Los alumnos poseen habilidades deseables para desarrollarse como miembros de un equipo de trabajo:

La mayoría de sus miembros:

O Coordina sus actividades.

O Comunican sus ideas.

Escuchan a todos sus integrantes.

O Reconocen sus limitaciones y habilidades ante el grupo.

Reconocen que existen deficiencias en el proceso de planificación de las reuniones de trabajo.

8. Los alumnos reconocen la diferencia entre grupo y equipo.

9. Más de la mitad de los entrevistados considera que su grupo no está actuando como un equipo.

10. Los estudiantes reconocen que una reunión de trabajo grupal no es planificada y sujeta a una estricta agenda.

11. Los alumnos reconocen que el trabajo en grupo es importante para su formación profesional.

\section{SUGERENCIAS.}

1. El numero ideal de integrantes para trabajar en grupo es cuatro para que funcione como un equipo, excepcionalmente puede formarse de cinco integrantes.

2. Los miembros que conforman un grupo, para ser considerados como equipos de trabajo, sus miembros deben:

O Escucharse unos a otros y las ideas deben respetarse.

O Asumir una responsabilidad conjunta.

O Fomentar el liderazgo compartido por varios.

O Discutir, decidir y trabajar de manera conjunta.

O Tomar decisiones por consenso, en el cual es evidente que todos, en general están de acuerdo $y$ dispuestos a adherirse.

O Repartirse el trabajo, tratando en lo posible de encontrar a la persona más adecuada para cada tarea. De hecho, el equipo establece un contrato social, entre sus distintos miembros, directamente ligado al propósito que se desea alcanzar.

3. Para lograr que un equipo sea efectivo, estos deben fijar:

O Visión compartida.

O Misión.

O Código de valores y principios guía del equipo.

O Un lugar de trabajo adecuado.

O Valorar las diferencias.

O Fomentar la responsabilidad compartida.

O Deben tener una metodología para sus reuniones de trabajo.

4. Para que un grupo funcione como equipo, debe:

O Fijarse objetivos concretos.

O Plasmar por escrito una agenda de trabajo $y$ ceñirse a ella.

- La discusión debe concentrarse en como deberá alcanzarse estos objetivos (estrategia).

Establecer como norma una comunicación fluida y eficaz.

O Fijar un programa de trabajo, con fechas, plazos y actividades a desarrollar (incluir un diagrama de Gantt).

O Crear y desarrollar el suficiente grado de compromiso, por parte de los miembros del equipo.

Fijar reglas de comportamiento muy claras.

5. La Biblioteca Central de la Universidad Nacional Mayor de San Marcos cuenta con cinco diferentes ambientes, el principal de los cuales es su auditorio: además, dos pequeños auditorios de distinto tamaño para la realización de vídeo - conferencias, ya no de grandes eventos, sino las cotidianas, por ejemplo; la de un grupo de investigadores o de un profesor con sus alumnos. Otros dos ambientes con capacidades para entre 50 y 60 personas, también pueden ser utilizados diversos niveles de la biblioteca para grupos de trabajo o reuniones de pequeña magnitud. La modularidad de estos ambientes permite que se usen individualmente o como salas concurrentes de un solo acto

Estos ambientes dan ocasión para que los alumnos se ejerciten en su comportamiento como equipos de trabajo, en las investigaciones que les son conferidas, como parte de su formación profesional. 
6. La acción del equipo debe estar basada en el esfuerzo sincronizado de todos, al igual que un equipo de baloncesto.

7. Las instituciones de educación superior deben crear las condiciones de infraestructura necesarias (ambiente de trabajo) para el desarrollo de trabajos en equipo, además de proporcionar la tecnología apropiada (cañón multimedia, retroproyector, etc.).

8. La evaluación que realiza el docente en el aula debe estar orientada a calificar los trabajos de investigación como equipos y no como grupos. Para ello se deben tomar en cuenta si la mayoría de sus miembros:

O Coordina sus actividades.

O Comunican sus ideas (participación).

Osuchan a todos sus integrantes.

O Hacen uso de la planificación en sus reuniones de trabajo.
9. Durante muchas décadas se ha valorado el trabajo individual y la competencia. Durante el colegio y la universidad, nos acostumbraron a notas individuales, ha llegado el momento de promover que los profesores sean guías y facilitadores en la formación de equipos, asesorándolos en el avance de la tarea, como en el desarrollo del proceso de grupo a equipo.

10. Capacitar a los docentes en un taller de equipos de trabajo y como llegar a ser guías y facilitadores de estos equipos. Trabajar en equipo adoptar una actitud de servicio, dejar de pensar sólo en nuestro beneficio. De esta forma contribuiremos a formar verdaderos equipos de trabajo.
1 Tomado de Douglas McGregor, The Human of Enterprise, Pág. 232-35. Copyright McGraw-Hill Books Company citado con la autorización de McGraw-Hill Bokk Company.

2 Rensis Likert, New Patterns of Management, pag. 166-169. Copyright. 1961 por McGraw-Hill Book Company. Citado con autorización de McGraw-Hill Book Company.
3 Robert Blake y Mouton, La Red Gerencial, Editorial Diana, 1985. España.

4 William G. Dyer. "Formación de equipos: Problemas y alternativas". Addison-Wesley Iberoamericana $y$ Sistema Técnico de Edició n. 2da. Edición, 1998. 


\section{B I B L I O G R A F I A}

1. DYER, WILLIAM G.

Formación de equipos: Problemas y alternativas. Iberoamericana y Sistema Técnico de Edición. Addison-Wesley.

2da. Edición, 1998.

2. JAVIER GARCIA RADA.

El Comportamiento Humano en las Organizaciones. Universidad del Pacifico. 1ra Edición, 1992. Lima.

3. MIKE ROBSON.

Cómo resolver problemas en equipos de trabajo autorregulados. Panorama Editorial. 1995. México.

\section{STEPHEN ROBBINS}

Comportamiento organizacional - Teorla y Práctica. Prentice Hall Hispanoamericana S.A. 1996, 7ma. Edición.

5. Revista Calidad y Excelencia, Año 2 Número 8

6. Gerencia Pública, Año 1 Número 3

Trabajando en equipo

7. Harvard Deusto Bussiness Review, 1996 Número 73 Equipos de Elite.
(*) Lic. en Administración. Egresado de la Maestria de Administracion UNMSM: Docente Asociado de la Facultad de Ciencias Administrativas. Autor de Artlculos y Manuales sobre su especialidad. Jefe de la Oficina de Planificación, Racionalización y Presupuestos y Miembro del Instituto de Investigaciones de la Facultad de Ciencias Administrativas UNMSM.
(*)

Equipo de Investigación

Planeamiento y Dirección: Lic. Luis Flores Hidalgo

Asistente Investigación: Juan Carlos Pacheco

$\begin{array}{ll}\text { Integrantes: } & \text { Juan Bardales Rojas } \\ & \text { Jorge Cabrera Montenegro } \\ & \text { Tatiana Miranda López } \\ & \text { Silvia San Miguel Pino } \\ & \text { Pedro Vera Loayza } \\ & \text { Patricia Vidal Pérez }\end{array}$




\section{A N EXO}
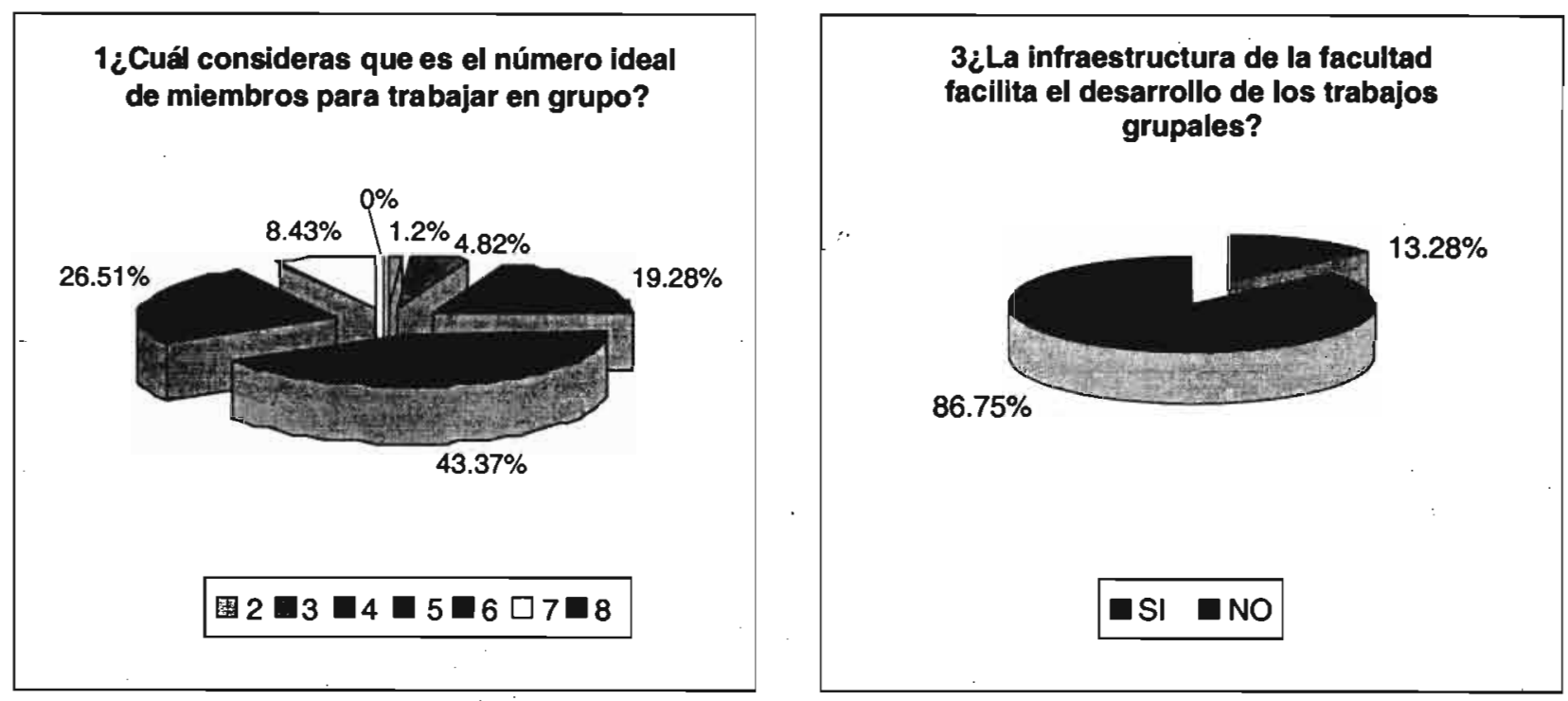

$2 i$ Consideras que el trabajo en grupo es importante en tu formación profesional?

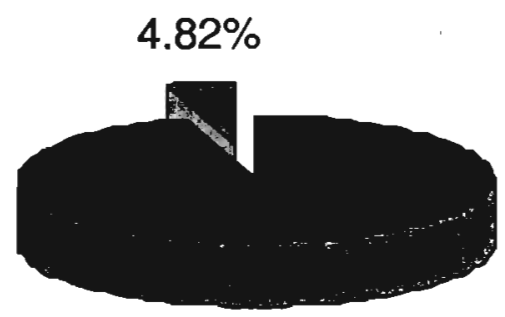

$95.18 \%$

$\square \mathrm{SI}$ NO
4. Si tu respuesta anterior fue negativa ¿cuáles consideras deberian ser las mejoras?

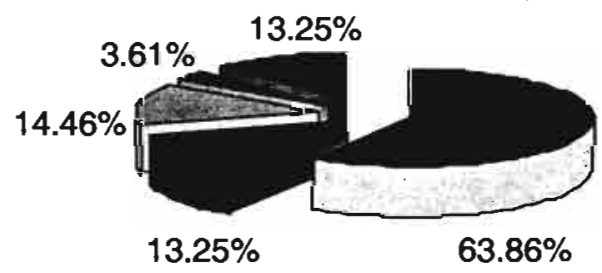

Amb ien tes pa trab ajos gr up ale

Mejo ra r la Biblio te ca

圈 Tecn olo gi a/ Equ ipos

Ase so ra mie nt o

No op ina 
5iConsideras que hay deficiencias en el proceso de las reuniones de trabajo grupal?

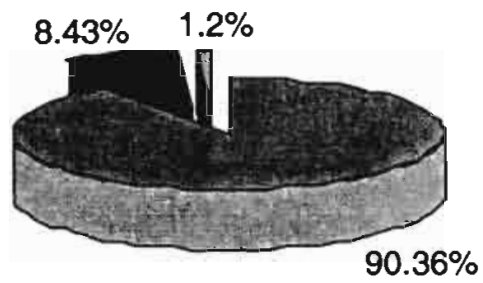

:

\section{$6 ¿$ Tiene un grupode metodologíapara} las reuniones de trabajo?

$57.83 \%$

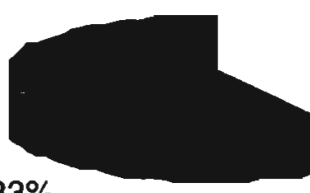

$42.17 \%$

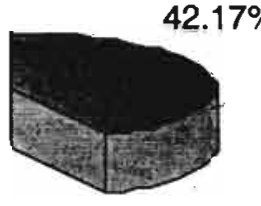

:SI $\square \mathrm{NO}$

\section{7¿Qué factores impiden el desarrollo efectivo de tu grupo?}

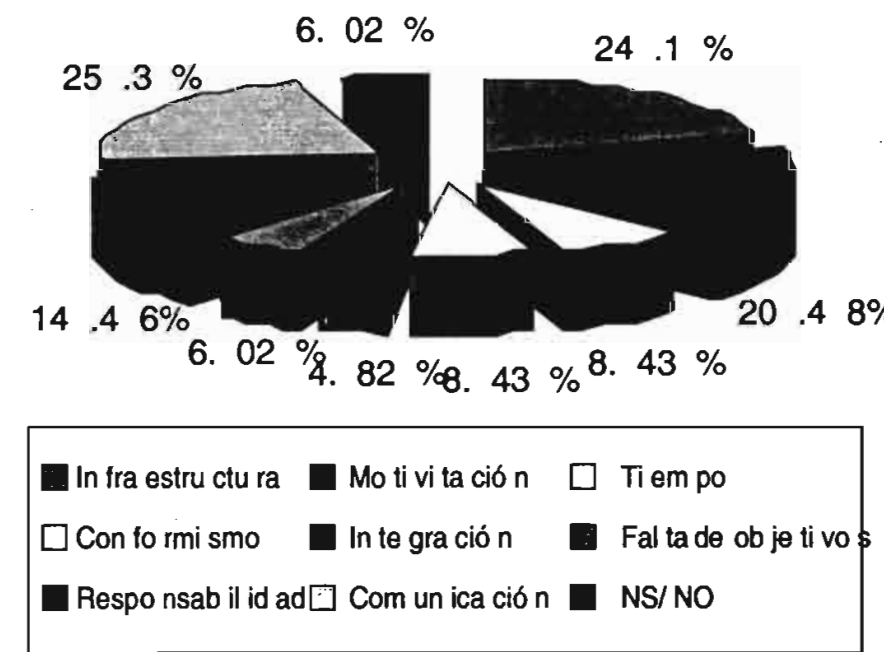

